\title{
The Search Engine of Image-Based High-Level Semantic Information with Application in Mobile E- Commerce
}

\author{
Yu Zhou', Zhiyuan Fang ${ }^{2}$, Yueqi Chen ${ }^{1}$, Ning $\mathrm{Li}^{1}$ and Yuanting $\mathrm{Ge}^{1}$ \\ 'Department of Computer Science, School of Information Science \& Engineering, Lanzhou \\ University, Lanzhou 730000, Gansu, P.R. China \\ yu.ioey.zhou@gmail.com chenyueq i2660@sina.com \\ ${ }^{2}$ School of Business, Sun Yat-sen University, Guangzhou 510080, P.R. China \\ drfang@dr.com
}

\begin{abstract}
In this paper, based on the Mobile E-commerce Platform, we implement the Information Retrieval System of image-based high-level semantic by using the feature extraction algorithm based on object semantic. By way of optimizing the image feature extraction algorithm, improving the structure of the traditional Search Engine and increasing the carrying capacity of mobile terminals, we will solve a core issue why Mobile E-commerce is not rife currently. It has profound effect to increase the probability of transaction, excavate potential customers and better the level of personalized service. At the same time, it satisfies the requirements of most customers.
\end{abstract}

Keywords: E-Commerce, Electronic market place, Search engine

\section{INTRODUCTION}

In Mobile E-commerce, people communicate with each other by mobile terminals and mobile communications networks or mobile communications networks and Internet E-commerce. Phone, PDA and other mobile equipments have become indispensable tools, that means that a sound environment for developing Mobile Ecommerce has been formed. Search Engine is one of the most important marketing tools. Traditional Search Engine Model can not satisfy the demands of consumer in the Mobile E-commerce application, in order to afford the convenience to our customers as much as possible and increase potential trading opportunities, how to improve the efficiency of search engines has been concerned by many e-commerce websites.

In this paper, we consider it from three aspects to propose an improved Search Engine of image-based high-level semantic information: the limitations of cell phones, the needs of e-business application and the characteristics of the Peer-to-Peer Network.

Please use the following format when citing this chapter:

Zhou, Y., Fang, Z., Chen, Y., Li, N., Ge, Y., 2007, in IFIP International Federation for Information Processing, Volume 255, Research and Practical Issues of Enterprise Information Systems II Volume 2, eds. L. Xu, Tjoa A., Chaudhry S. (Boston: Springer), pp. 825-830. 


\section{IMPROVED SEARCH ENGINE MODEL}

\subsection{The Search Engine Entire Framework}

The Search Engine mainly runs on the central server, mobile clients communicate indirectly with e-commerce websites by using the central server. Traditional Search Engine Model can not satisfy the demands of consumer in the Mobile E-commerce applications, so we need to come up with a new model. Based on the model, we can use pictures in the phone instead of keywords to query.

Compared with the conventional Search Engines, in this paper, images inputted by Users are not retrieved directly, but after accepted and then extracted feature information as matching information through image processing module. It can reduce matching information, improve the retrieval speed and matching accuracy in doing so. In short, image processing is the key in the Search Engine. We will introduce an improved Search Engine Structure in Fig.1.

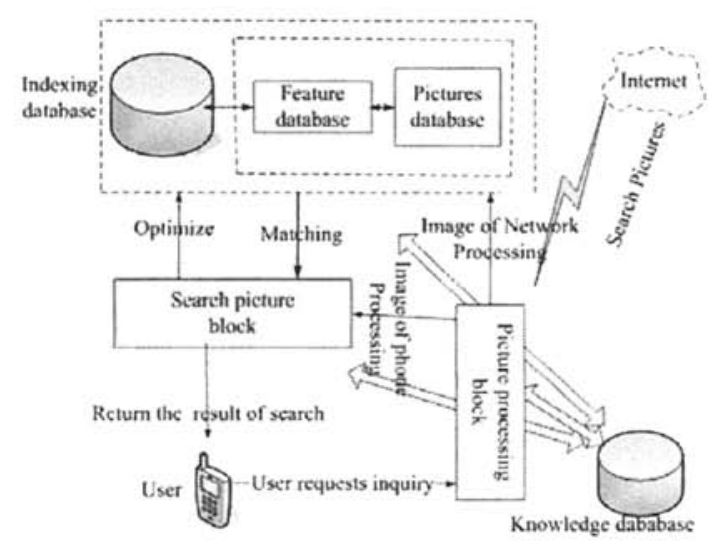

Figure 1. Search Engine Model

\subsection{Graphics Processing Model Design}

Our Graphics Processing Model uses mainly the way based on image high-level semantic information retrieval. Having analyzed most images in the taobao website, we find most images have some common characteristics: 1. Prominent position of images; 2 . Most images are in the middle; 3 . Light obviously; 4 . Images have much background information.

With comprehending these features of images, we analyze the images album and find object-based semantic retrieval is the most appropriate way in several high-level semantic layers. According to the feature information, we establish image index. The amount of information affects directly the processing speed of the entire Search Engine. The less information the images have, the better the performance the Search 
Engine has. Thus, the search of image-based semantic layer filters much background information and improves the system's whole performance. Meanwhile, the indexes are established according to the characteristics information of objects.

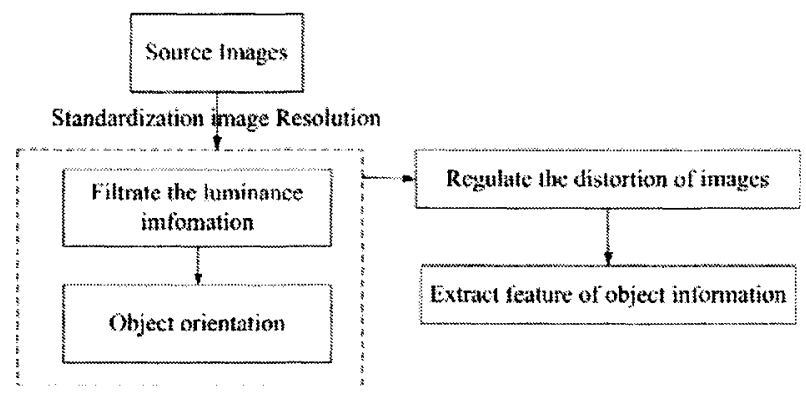

Figure 2. Feature Extraction Process Object-based Semantic of Images

After object orientation, we extract feature information of images by using the existing methods and techniques, store it into feature database, and then establish Index vectors. At last, we made the image processing module of image-based processing. The image processing module is designed specifically and shown in figure 2.

In this paper, Image Processing Module is the key to achieve the query and improve the performance, and it is also the focus of this paper.

\section{OBJECT-BASED SEMANTIC FEATURE EXTRACTION}

\subsection{The Feature Extraction Method of Object-based Semantic}

Most commodities of the hardware and building materials have smooth texture and obvious shape. Thus, the method of combination of shape and texture has comparative advantage in image processing, however, the character of texture is no orderliness, some have relatively smooth texture, but others' gradient in texture change rather largely. If we use the same method as building materials and hardware products to process image, the error will be rather big. Therefore, we analysis and find that this combination can extract characteristics of most images, combining color, texture and shape, and we sort the different merchandise images and give different weights to each species. Obvious features are given heavy weight, not obvious features are given light weights in the same kind images of merchandise. Calculated Formula is shown as Formula 1.

$$
s=\frac{w_{1} s_{c}+w_{2} s_{w}+w_{3} s_{p}}{w_{1}+w_{2}+w_{3}}
$$

$w_{1}, w_{2}, w_{3}$ is feature weight, $s_{c}$ is color feature, $s_{w}$ is texture feature, $s_{p}$ is shape feature. 


\subsection{The Feature Extraction Algorithm of Object-based Semantic}

\subsubsection{Feature Extraction Process}

The algorithm of feature extraction currently doesn't preprocess the luminance information, but only processes image directly. In the paper, we filter the luminance information which interferes with feature extraction and just retain color information. We choose mainly an existing feature extraction algorithm to balance the speed and the accuracy based on three characters of color, texture and shape. The steps of implementation are as follows:

Input an image, and then adjust to the same Resolution and size.

Preprocess the luminance information of the image.

Regulate the distortion of the image.

Give different weights to different features including color, shape, and texture to process different classifications of merchandise images, position the object, and extract the feature information within the range of the object at the same time.

Store the extracted feature information, store the address information in index database, and set up an index for vectors.

\subsubsection{Feature Transformation}

To give prominence to details, the most commonly used method of traditional image processing is gradient processing. According to the field theory, given a function $f(x, y)$, the gradient of point $(x, y)$ can be defined as a vector, namely gradient vector as formula 2 .

$$
\operatorname{grad}[f(x, y)]=\left[\frac{\partial f}{\partial x}, \frac{\partial f}{\partial y}\right]^{T}
$$

The images on the Internet are discrete scalar images, so we need to scalarize the vector information of the formula. We use formula 3 to scalarize the vector information.

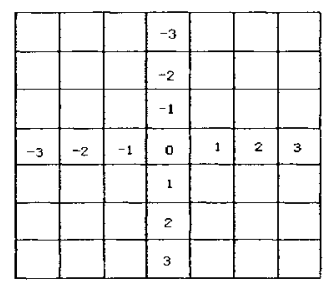

Figure 3. 7 *7 Block Area of the Image

The gradient of a hue vector can be calculated by the first feature space conversion. As shown in Figure 3, in an image region of $7 * 7$, every square indicates to a pixel of the image. The row number is $\mathrm{i}$ and the column number is $\mathrm{j}$.

The scalar function $\mathrm{G}_{\mathrm{f}(\mathrm{x} . \mathrm{y})}$ is used to calculate the hue vector gradient of the central point. 
The Search Engine of Image-Based High-Level Semantic Information with Application in

$G_{f(x . y)}=\max \{\operatorname{Dis}(\operatorname{Color}(x, y), \operatorname{Color}(x+i, y+j))$ where $i=-3,-2,-1,0,1,2,3 ; j=-3,-2,-1,0,1,2,3\} \quad(3)$

Here, Color $(x, y)$ is the hue vector of the coordinate $(x, y)$, the function Dis (Colorl, Color2) represents the distance of Color 2 from Color1. Color1 and Color2 are hue vectors.

$\mathrm{CT}(\mathrm{x}, \mathrm{y})$ represents a 2-dimensional feature variable from the feature space after the first feature space transformation of the original color image. Each eigenvalue indicates the gradient of a color vector on the coordinate $(x, y)$. The first feature change is subject to calculating the hue gradient. After that, the luminance information and color information distributes aslant. So future processing of $\mathrm{CT}(\mathrm{x}, \mathrm{y})$ is still required for achieving the final effect. It's the secondary transformation.

Here we use the formula 4 of secondary transformation.

$$
\lambda(i, j)=\sum_{R q=1}^{n-1} \frac{\operatorname{sum}-\operatorname{ring}(R q)_{(i, j)}}{8 R q}+C T(i, j)
$$

\section{ANALYSIS OF TEST RESULT}

There are two methods to extract the image features. One is to extract after filtering the luminance information, the other is not. We compare the two methods.

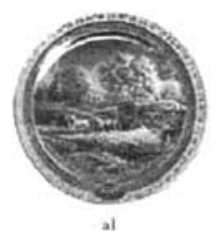

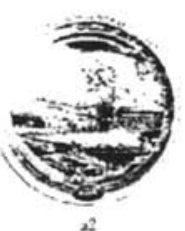

22

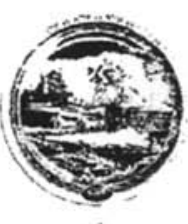

$3^{3}$

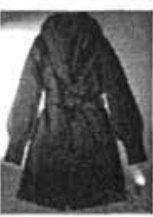

b1

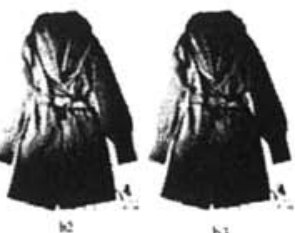

b3

Figure 4. Comparative Results

a1, b1 are resource images, a2, b2 are images processing directly, a3, b3 are image processing after preprocess the luminance information. From the test results, we can see that the luminance information as noise gives negative effects on extracting features. And it's clear that the feature extracting is better after filtering the luminance information.

We test 50 groups of images which come from Baidu. All programs have been implemented in Microsoft Visual $\mathrm{C}++6.0$, and parts of codes are provided by [4].

\section{REFERENCES}

1. D. Cheng and D. Cui, Design of an efficient system for mobile E-commerce, Journal of Computer Applications. Volume 2, Number 2, pp.515-516, (2006).

2. N. Wu and F. Song, A kind of Image searches based on the semantic information, Chinese Journal of Image and Graphics. Volume 12, Number 11, pp.1774-1780, (2006). 
3. X. Fan, X. Xie, and Z. Li, Photo-to-Search: Using Multimodal Queries to Search the Web from Mobile Devices, in Proc. of the 7th ACM SIGMM international workshop on Multimedia information retrieval (2005), pp.143-150.

4. B. Jian, Visual C++ Digital Image Processing (People's Posts \& Telecommunications Publishing House: Beijing, 2002). 